\title{
Kerker effect, superscattering, and scattering dark states in atomic antennas
}

\author{
Rasoul Alaee $\odot,{ }^{1,2,3, *}$ Akbar Safari, ${ }^{1,3}$ Vahid Sandoghdar, ${ }^{2,3,4, \dagger}$ and Robert W. Boyd $\odot^{1,3,5}$ \\ ${ }^{1}$ Department of Physics, University of Ottawa, 25 Templeton Street, Ottawa, Ontario K1N 6N5, Canada \\ ${ }^{2}$ Max Planck Institute for the Science of Light, D-91058 Erlangen, Germany \\ ${ }^{3}$ Max Planck-University of Ottawa Centre for Extreme and Quantum Photonics, 25 Templeton Street, Ottawa, Ontario K1N 6N5, Canada \\ ${ }^{4}$ Department of Physics, Friedrich Alexander University Erlangen-Nuremberg, D-91052 Erlangen, Germany \\ ${ }^{5}$ The Institute of Optics, University of Rochester, Rochester, New York 14627, USA
}

(Received 10 August 2020; accepted 30 November 2020; published 22 December 2020)

\begin{abstract}
We study scattering phenomena such as the Kerker effect, superscattering, and scattering dark states in a subwavelength atomic antenna consisting of atoms with only electric dipole transitions. We show that an atomic antenna can exhibit arbitrarily large or small scattering cross sections depending on the geometry of the structure and the direction of the impinging light. We also demonstrate that atoms with only an electric dipole transition can exhibit a directional radiation pattern with zero backscattering when placed in a certain configuration. This is a special case of a phenomenon known as the Kerker effect, which typically occurs in the presence of both electric and magnetic transitions. Our findings open a pathway to design highly directional emitters, nonradiating sources, and highly scattering objects based on individually controlled atoms.
\end{abstract}

DOI: 10.1103/PhysRevResearch.2.043409

\section{INTRODUCTION}

Scattering is a fundamental phenomenon in light-matter interaction, and the scattering cross section is a measure to describe how strongly an object interacts with incident photons [1]. The ability to manipulate and control scattering is an important research goal in optics and atomic physics. In nanophotonics, the scattering can be controlled by engineering the geometry of the optical antennas as the canonical elements of the interaction [2]. In particular, it has been shown that engineered nanoparticles can exhibit remarkable scattering phenomena such as directional radiation patterns with zero backscattering known as the Kerker effect [3-6], the scattering dark state [7-9], and superscattering [10-12]. To observe these phenomena, the nanoparticles are designed to support not only an electric dipole but also a magnetic dipole and higher-order multipoles. The underlying physics of these scattering phenomena can be understood from the interference among the scattered fields of all the induced multipole moments [3-17].

With the recent developments in manipulating and controlling single atoms and quantum emitters, a new class of subwavelength antennas consisting of quantum emitters have been employed in the past decades to control light-matter

\footnotetext{
*Corresponding author: rasoul.alaee@gmail.com

${ }^{\dagger}$ vahid.sandoghdar@mpl.mpg.de
}

Published by the American Physical Society under the terms of the Creative Commons Attribution 4.0 International license. Further distribution of this work must maintain attribution to the author(s) and the published article's title, journal citation, and DOI. Open access publication funded by the Max Planck Society. interaction at the atomic scale [18-41]. Here, it should be borne in mind that atoms arranged in a subwavelength structure interact with the radiation field cooperatively [42-45]. Hence, although the canonical elements of these systems remain atoms with only electric dipole transitions, it turns out that the entire structure can be modeled as a single antenna that effectively exhibits both electric and magnetic multipoles $[38,39]$ and thus enables the control of light scattering (see Fig. 1).

In this paper, we treat the situation of a few atoms in simple geometries and show that the scattering of the structure can be significantly different from the scattering of the individual atoms (see Fig. 1). Each atom supports only an electric dipole transition with a maximum scattering cross section of $3 \lambda^{2} / 2 \pi$ [46-49]. Using the multipole expansion [14-16], the effective electric and magnetic multipole moments of the atomic antenna can be found from the induced current $\mathbf{J}(\mathbf{r}, \omega)$. By employing these multipole moments, we show in the following that it is possible to tailor the scattering of the structure to achieve extremely large or small scattering cross sections (the so-called superscattering and scattering dark state, respectively), as well as the Kerker effect. Our proposed antennas can be realized experimentally using the available experimental methods in cold atom manipulation $[37,40]$.

\section{ATOMIC ANTENNA IN THE WEAK-EXCITATION LIMIT}

The atoms in Fig. 1 are modeled as two-level systems and are arranged such that the overall size of the antenna is smaller than the wavelength of the incident light. We assume an isotropic and linear atomic response, i.e., a weak excitation such that the atomic transition is far below the saturation limit. 


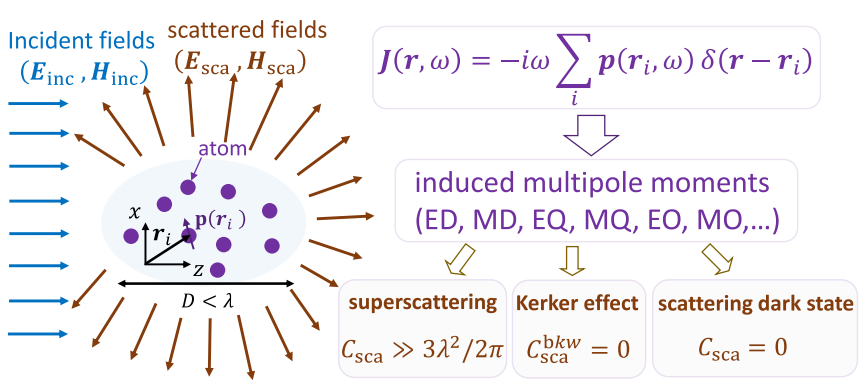

FIG. 1. Main idea of this work. Sketch of an atomic antenna composed of a few atoms with only electric dipole transition moments. The overall size of the antenna $D$ is smaller than the wavelength of light. The induced polarization current $\mathbf{J}(\mathbf{r}, \omega)$ of the antenna can be found from the induced dipole moment of the atoms, $\mathbf{p}\left(\mathbf{r}_{i}\right)$ placed at $\mathbf{r}_{i}$. The induced current is decomposed into multipole moments which are used to control the scattering cross section of the structure. The excitation and the position of the atoms can be tailored to achieve arbitrary induced electric and magnetic multipoles. Thus it is possible to control the scattering and achieve scattering dark states, superscattering, and the Kerker effect. The maximum scattering cross section of a single atom, $3 \lambda^{2} / 2 \pi$, is considered as the measure.

Therefore the electric polarizability of each atom amounts to $\alpha(\omega)=-\left(\alpha_{0} \Gamma_{0} / 2\right) /\left(\delta+i \Gamma_{0} / 2\right)$, where $\alpha_{0}=6 \pi / k^{3}$ ( $k$ is the wave number of the resonant light), $\Gamma_{0}$ is the radiative decay rate of the atomic transition at frequency $\omega_{a}$, and $\delta=$ $\omega-\omega_{a} \ll \omega_{a}$ represents the frequency detuning between the illumination and the atomic resonance $[47,50]$. The antenna is illuminated by a plane wave $\mathbf{E}_{\mathrm{inc}}(\mathbf{r})=E_{0} e^{i \mathbf{k} \cdot \mathbf{r}} \mathbf{e}_{E}$ propagating in the $\mathbf{k}$ direction, where $\mathbf{e}_{E}$ is the unit vector in the electric field direction and $E_{0}$ is the electric field amplitude. The extinction cross section is defined as

$$
C_{\text {ext }}=\frac{k}{\epsilon_{0}\left|E_{0}\right|^{2}} \sum_{i=1}^{N} \operatorname{Im}\left[\mathbf{p}\left(\mathbf{r}_{i}\right) \cdot \mathbf{E}_{\mathrm{inc}}^{*}\left(\mathbf{r}_{i}\right)\right],
$$

where $*$ indicates the complex conjugate and $\mathbf{p}\left(\mathbf{r}_{i}\right)$ is the induced dipole moment of the $i$ th atom. The optical theorem indicates that for atoms without any nonradiative transitions, the extinction and scattering cross sections are identical, i.e., $C_{\text {sca }}=C_{\text {ext }}$ [47,51]. The dipole moment of the atoms can be calculated from the following coupled-dipole equations [52,53]:

$$
\mathbf{p}\left(\mathbf{r}_{i}\right)=\epsilon_{0} \alpha\left(\mathbf{E}_{\mathrm{inc}}\left(\mathbf{r}_{i}\right)+\sum_{j=1, j \neq i}^{N} \mathbf{G}\left(\mathbf{r}_{i}, \mathbf{r}_{j}\right) \mathbf{p}\left(\mathbf{r}_{j}\right)\right),
$$

where $\mathbf{G}\left(\mathbf{r}_{i}, \mathbf{r}_{j}\right)$ is Green's tensor [54,55]. Equation (2) can be solved numerically for an arbitrary geometry of the atoms and the driving field. We note that this theoretical model captures the essence of the experimental results for cold atomic arrays [37].

\section{SUPERSCATTERING}

In general, the maximum scattering cross section of an isotropic scatterer is $C_{\mathrm{sca}, j}^{\max }=(2 j+1) \frac{\lambda^{2}}{2 \pi}$, where $j$ is the order of the multipole; e.g., $j=1,2$, and 3 , for dipoles,

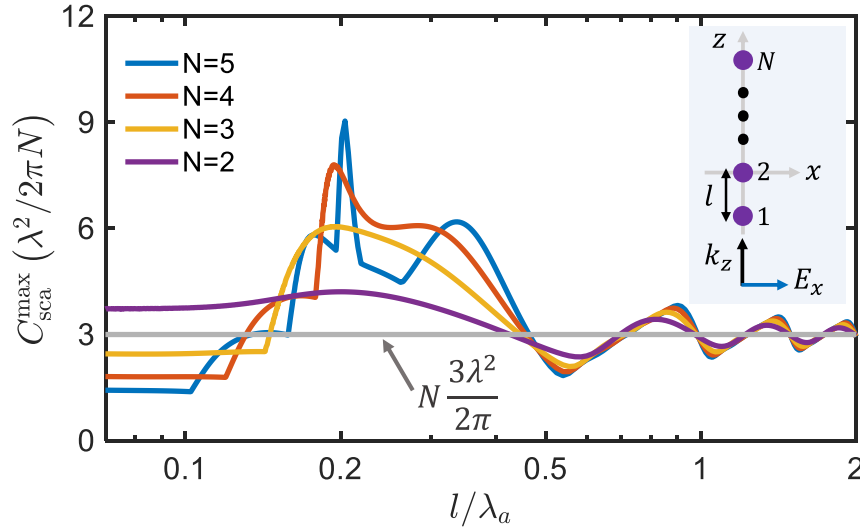

FIG. 2. Superscattering. Maximum scattering cross section of $N$ atoms normalized to $\lambda^{2} / 2 \pi$ as a function of the distance between the atoms $l$. The gray line shows the maximum scattering cross section for $N$ noninteracting atoms, i.e., $N \times \frac{3 \lambda^{2}}{2 \pi}$. The geometry of the atoms and the driving field is shown in the inset. The maximum scattering occurs at different frequency detunings for each $l$.

quadrupoles, and octupoles, respectively [10,11]. For example, the maximum scattering cross section of a single dipolar scatterer (such as the two-level atom considered in this paper) occurs at the resonance frequency and is limited to $3 \lambda^{2} / 2 \pi$ $[48,56]$. However, it is possible to engineer the structure of the scatterer to align the resonance frequencies of different multipoles and thus enhance the scattering cross section dramatically $[10,11]$. Here, we show that a particular arrangement of $N$ atoms can exhibit a scattering cross section significantly larger than $N \times \frac{3 \lambda^{2}}{2 \pi}$, although each individual atom supports only an electric dipole transition.

Consider $N$ atoms equally placed on the $z$ axis and illuminated by an $x$-polarized plane wave propagating in the $z$ direction as shown in the inset of Fig. 2. By using Eqs. (1) and (2), we plot the normalized maximum scattering cross section as a function of the distance between the atoms $l$. At large atomic separations, i.e., $l>\lambda_{a}$, the atoms are nearly noninteracting, and the maximum cross section is limited to $N \times \frac{3 \lambda^{2}}{2 \pi}$ (see the gray line in Fig. 2). However, the atomic antenna exhibits superscattering at one particular atomic spacing, $l \approx 0.2 \lambda_{a}$. Notably, the maximum scattering cross section for $N=5$ is approximately $15 \times \frac{3 \lambda^{2}}{2 \pi}$. We note that an even larger scattering cross section for a subwavelength atomic antenna can be achieved with a three-dimensional (3D) atomic arrangement (see Appendix B for details).

In order to understand the nature of this enhancement in the scattering cross section, we examine the case of an atomic tetramer with $N=4$ [see the inset of Fig. 3(a)]. The normalized scattering cross section as a function of frequency detuning and distance $l$ is shown in Fig. 3(a). The tetramer exhibits four modes [Fig. 3(b)]. $M_{1}$ is a superradiant mode, where all dipoles oscillate in phase, and exhibits a large linewidth compared with the natural linewidth of an isolated atom [25,42-45]. In contrast, $M_{4}$ is subradiant with a very narrow linewidth.

Figure 3(c) shows the normalized scattering cross section as a function of frequency detuning for $l=0.2 \lambda_{a}$ and the contribution of each multipole moment to the 

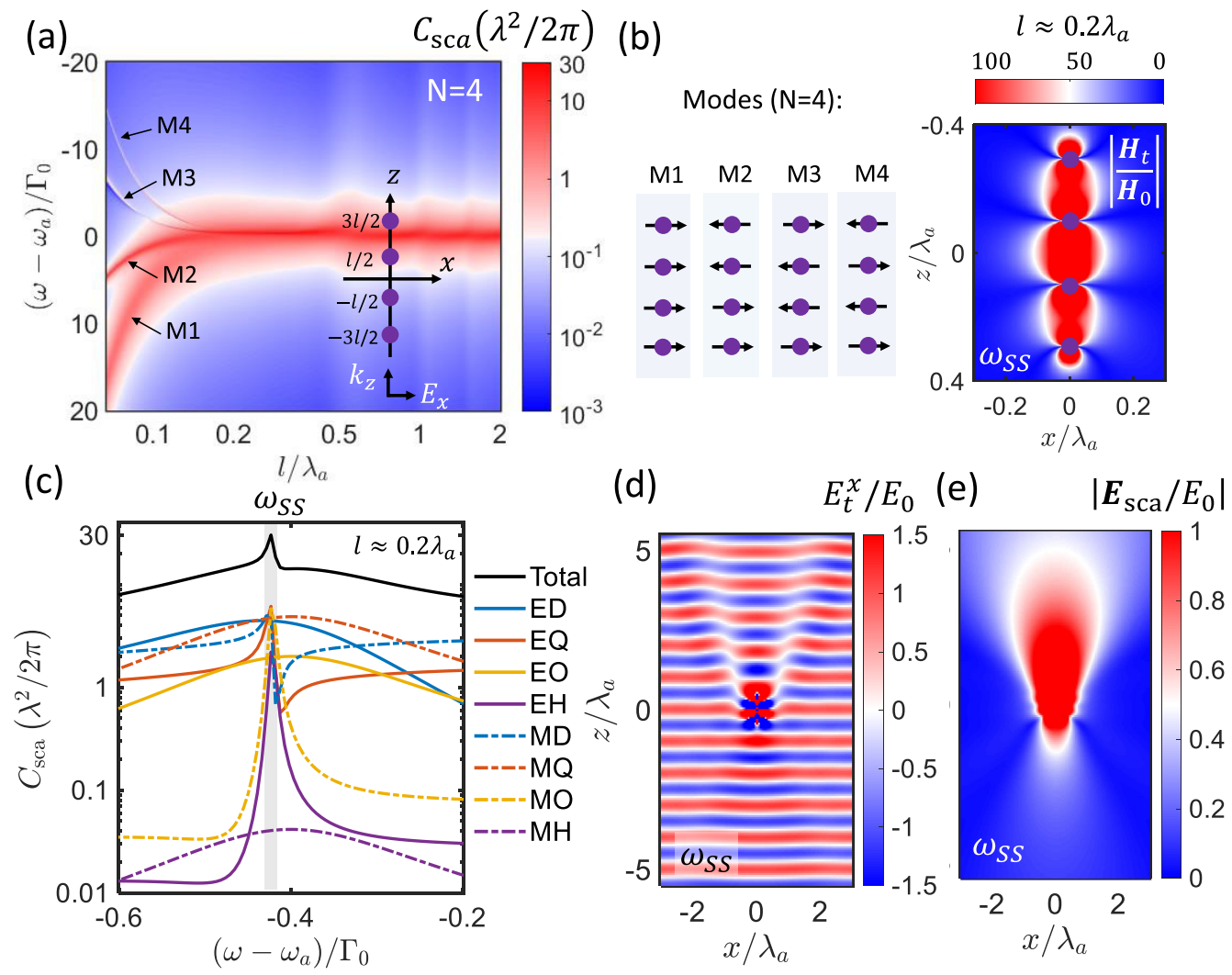

FIG. 3. Superscattering in a tetramer. (a) Scattering cross section normalized to $\lambda^{2} / 2 \pi$ and shown in logarithmic scale. Inset: illustration of an atomic tetramer placed at $\mathbf{r}_{1,2}= \pm l / 2 \mathbf{e}_{z}, \mathbf{r}_{3,4}= \pm 3 l / 2 \mathbf{e}_{z}$. (b) The tetramer exhibits four modes, $M_{1}$ to $M_{4}$. Total magnetic field distribution $\mathbf{H}_{t}$ at $\omega_{\mathrm{SS}}$, normalized to the incident magnetic field $H_{0}$. (c) Normalized scattering cross section of different electric and magnetic multipole moments as a function of frequency detuning for $l \approx 0.2 \lambda_{a}$. ED (MD), EQ (MQ), EO (MO), and EH (MH) indicate the electric (magnetic) dipole, quadrupole, octupole, and hexadecapole moments, respectively. (d) and (e) Real part of the normalized total electric field distribution $E_{t}^{x}$ (the $x$ component) and normalized scattered field distribution $\left|\mathbf{E}_{\mathrm{sca}}\right|$ at $\omega_{\mathrm{SS}}$, respectively (see the angle-dependent scattered power in Appendix B).

scattering cross section. Remarkably, the maximum scattering cross sections of different multipoles occur approximately at the same frequency, which is labeled by $\omega_{\text {SS }}$ in Fig. 3(c). The total scattering cross section is a superposition of the cross sections associated with effective electric and magnetic dipole, quadrupole, and octupole moments. Therefore the scattering cross section of the tetramer far exceeds the scattering cross section of a single atom. The strong magnetic field in Fig. 3(b) testifies to the induced magnetic multipolar response at $\omega_{\mathrm{SS}}$. Using $\mathbf{E}_{\text {sca }}(\mathbf{r})=\sum_{i=1}^{N} \mathbf{G}\left(\mathbf{r}, \mathbf{r}_{i}\right) \mathbf{p}\left(\mathbf{r}_{i}\right)$, the scattered and the total (sum of incident and scattered) fields of the atomic antenna in free space can be calculated at r. Figures 3(d) and 3(e) show the scattered and total field distribution at the superscattering frequency $\omega_{\text {SS }}$. The scattering field is very large even at the far field of the tetramer $(\gg \lambda)$, and the total field is strongly perturbed [Fig. 3(d)]. Therefore a higher extinction can be achieved compared with that of a single atom [48,57].

\section{SCATTERING DARK STATES}

While each atom is excited close to the resonance and scatters the photons individually, it is possible, in some certain conditions, to achieve a negligible overall scattering from the structure. This phenomenon, known as the scattering dark state (SDS), has been investigated in nanoparticles [7-9]. In an
SDS, the current distribution, and hence the electromagnetic field, is nonzero or even very large in the vicinity of the structure. However, similar to a nonradiating source [7], the current distribution does not radiate to the far field. Therefore such a structure becomes invisible under the conditions of an SDS [9]. Here, we study scattering dark states in an atomic antenna with a nonzero induced current and very large near fields. Consider an atomic trimer placed on the $x$ axis and illuminated by an $x$-polarized plane wave propagating in the $z$ direction [shown in the inset of Fig. 4(a)]. The extinction cross section of the atomic trimer can be calculated using $C_{\text {ext }}=k \operatorname{Im}\left(\alpha_{\text {sum }}\right)$, where $\alpha_{\text {sum }}$ reads as (see Appendix C)

$$
\alpha_{\text {sum }}=\sum_{i=1}^{3} \alpha_{i}=\alpha \frac{\epsilon_{0} \alpha\left(\beta_{2}-4 \beta_{1}\right)-3}{\epsilon_{0} \alpha\left(2 \epsilon_{0} \alpha \beta_{1}^{2}+\beta_{2}\right)-1}
$$

and where $\alpha_{i}$ is the effective electric polarizability of each atom at its position. $\beta_{1}=G_{12}^{x x}(k l)$ and $\beta_{2}=G_{13}^{x x}(2 k l)$ are components of Green's tensor (see Appendix C). The normalized scattering cross section as a function of frequency detuning and distance between the atoms $l$ is plotted in Fig. 4(a). The trimer can support multiple modes. However, only two modes can couple to the driving plane wave. These modes are subradiant and superradiant modes $[18,19,21,22,24,25,42-45]$ and are labeled by $M_{1}$ and $M_{2}$ 

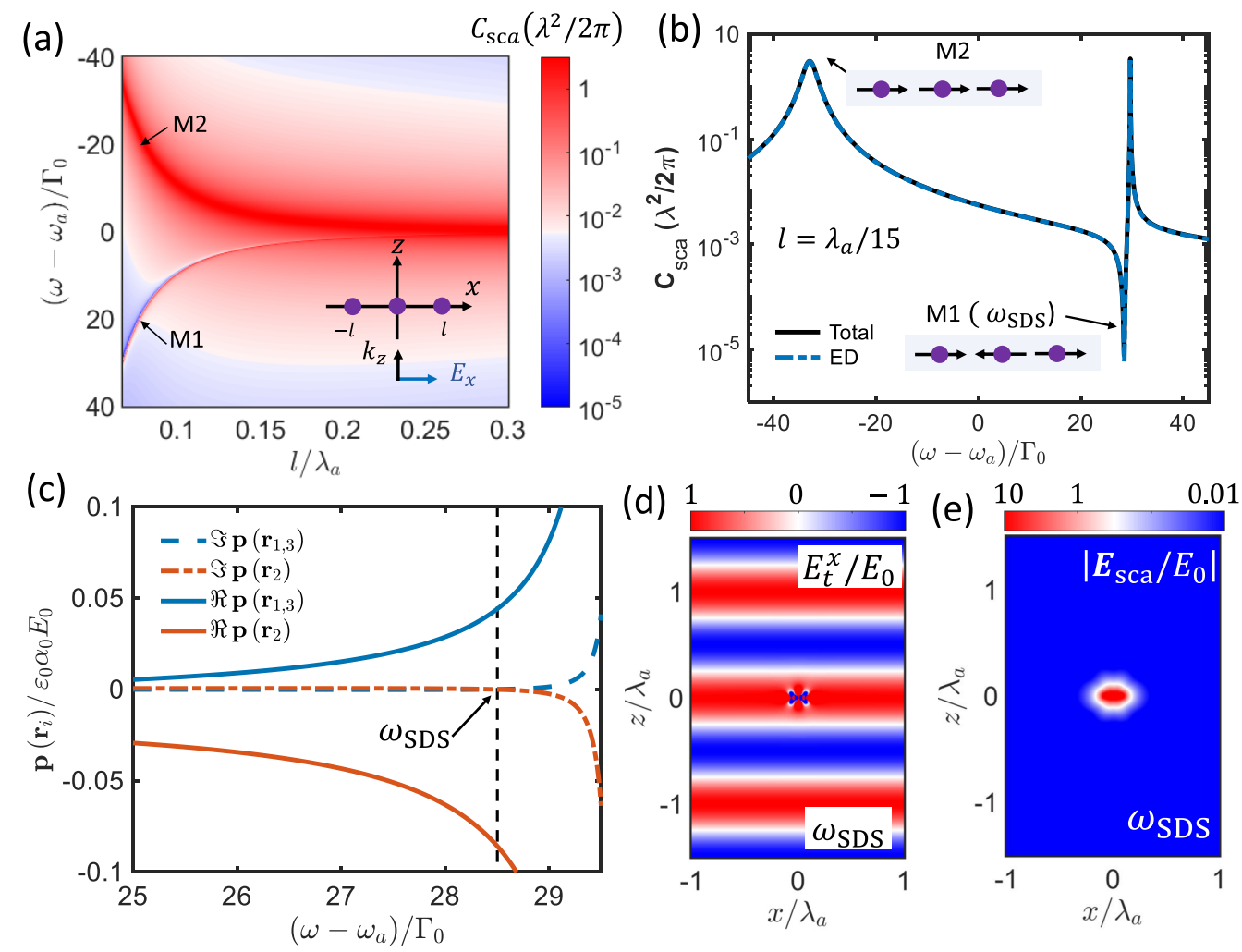

FIG. 4. Scattering dark state as a nonradiating source. (a) Scattering cross section (normalized to $\lambda^{2} / 2 \pi$ and shown in logarithmic scale) as a function of frequency detuning and the distance between the atoms $l$. Inset: schematic drawing of an atomic trimer composed of atoms at $\mathbf{r}_{1,3}=\mp l \mathbf{e}_{x}, \mathbf{r}_{2}=0$ and the plane-wave excitation. (b) Normalized scattering cross section as a function of frequency detuning for $l=\lambda_{a} / 15$. The dashed line shows the contribution of the electric dipole moment. The trimer exhibits two modes $M_{1}$ and $M_{2}$ when illuminating by a plane wave. (c) Real and imaginary parts of the induced dipole moment of each atom as a function of frequency detuning. At $\omega_{\text {sDS }}$, the individual atoms have nonzero dipole moments. (d) Real part of the normalized total field distribution $E_{t}^{x}$ at the frequency of the scattering dark state $\omega_{\text {SDS }}$. (e) Normalized scattered field distribution $\left|\mathbf{E}_{\mathrm{sca}}\right|$ at $\omega_{\mathrm{SDS}}$ (shown in logarithmic scale).

in Fig. 4, respectively. Figure 4(b) shows the normalized scattering cross section as a function of frequency detuning for $l=\lambda_{a} / 15$. At the scattering dark state, $\omega_{\mathrm{SDS}} \approx 28.5 \Gamma_{0}$, the trimer is transparent, and we observe that the scattering cross section is significantly suppressed by five orders of magnitude compared with the on-resonance excitation. Therefore the trimer becomes invisible to the incident field at the scattering dark state as the scattered field of the atoms interferes destructively in the far field. By using a multipole expansion of the induced current at $\mathbf{r}=0$, we show that the atomic trimer supports only an electric dipole moment, i.e., $p_{\text {eff }}^{x} \approx \epsilon_{0} E_{0} \alpha_{\text {sum }}$ [Fig. 4(b)], and all the higher multipoles are negligible (see Appendix C). Note that at $\omega_{\text {SDS }}$ all the effective multipole moments vanish, which is the condition for the SDS [7]. While the scattered field is nearly zero, the induced dipole of each atom and the induced current are not negligible as shown in Fig. 4(c).

At the transparency frequency $\omega_{\text {SDS }}$, we observe an extremely small perturbation to the incident plane wave [Fig. 4(d)]. Remarkably, the field distribution at $\omega_{\text {SDS }}$ implies that the electromagnetic field generated inside the scatterer is nonzero, and indeed, it is one order of magnitude larger than the incident electric field [Fig. 4(e)]. At $\omega_{\text {SDS }}$, the induced current of the trimer is a nearly nonradiating source. In Appendix $\mathrm{C}$, we investigate antennas consisting of a larger number of atoms. We show that for an antenna with $n$ excited modes there are $n-1$ scattering dark states. In other words, only one resonant mode radiates, while the other resonances do not radiate because of vanishing induced multipole moments [7,9]. This effect is reminiscent of the situation of optical cavities [58]. Note that the scattering dark states depend on the geometry of the atomic antenna and the polarization of the impinging light.

\section{KERKER EFFECT}

An atom or a scatterer with only an electric dipole transition has an omnidirectional radiation pattern, i.e., scatters lights in both forward and backward directions. However, a Huygens' scatterer, a scatterer with balanced electric and magnetic dipole moments, can exhibit a directional radiation pattern with zero backscattering. This effect is also known as the first Kerker condition [3]. The directional radiation with zero backscattering relies on constructive and destructive interference of the scattered field of the induced electric and magnetic dipole moments in the forward and backward directions, respectively. The Kerker condition has been studied in engineered high-index dielectric and metallic nanoparticles $[3,5]$. In the following, we show that an atomic trimer in an equilateral triangle configuration [see the inset of Fig. 5(a)] 

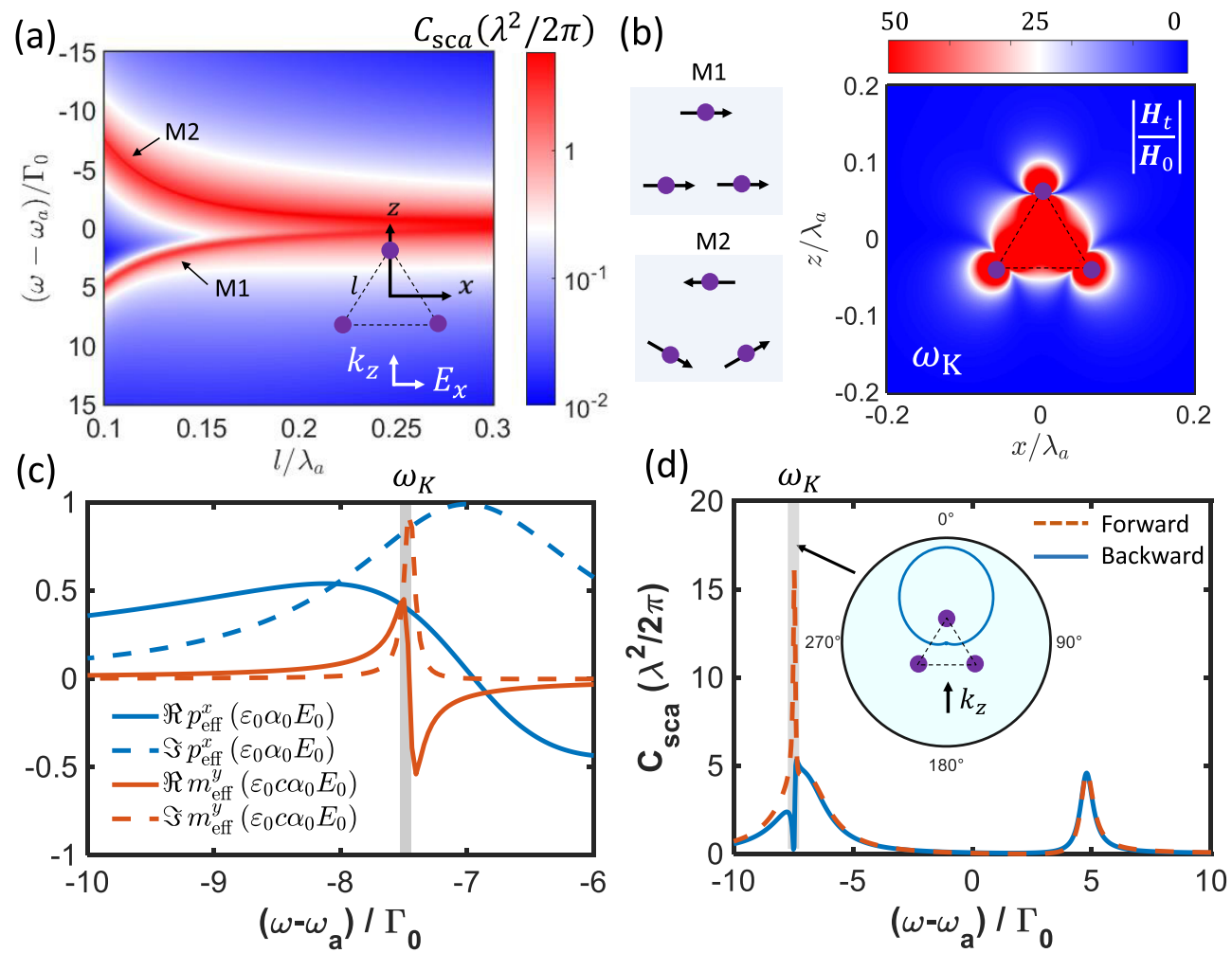

FIG. 5. Kerker effect. (a) Total scattering cross section normalized to $\lambda^{2} / 2 \pi$ as a function of the distance between the atoms. The inset illustrates an atomic trimer in an equilateral triangle composed of atoms at $\mathbf{r}_{1,2}= \pm l / 2 \mathbf{e}_{x}-\frac{\sqrt{3}}{6} l \mathbf{e}_{z}, \mathbf{r}_{3}=\frac{\sqrt{3}}{3} l \mathbf{e}_{z}$. (b) Normalized total magnetic field distribution at $\omega_{\mathrm{K}}$. The trimer exhibits two modes $M_{1}$ and $M_{2}$. (c) Real and imaginary parts of the induced electric $p_{\mathrm{eff}}^{x}$ and magnetic $m_{\text {eff }}^{y}$ dipole moments calculated from Eq. (4). The Kerker condition is met at $\omega_{\mathrm{K}}$ where the real and imaginary parts of the induced dipole moments satisfy the condition $p_{\text {eff }}^{x}=m_{\text {eff }}^{y} / c$, independently. (d) Normalized forward-scattering (red line) and backward-scattering (blue line) cross section at $l=0.1 \lambda_{a}$ calculated from Eq. (5). Inset: the radiation pattern at the Kerker condition, which shows zero backward scattering.

can satisfy the first Kerker condition, although the constituent atoms support only an electric dipole transition. The normalized scattering cross section as a function of frequency detuning and the distance between the atoms $l$ is shown in Fig. 5(a). The trimer exhibits two modes as shown with $M_{1}$ and $M_{2}$ in Fig. 5(b). We will show below that the $M_{2}$ mode exhibits a strong magnetic response induced by the electric current, i.e., $\mathbf{J}(\mathbf{r}, \omega)$ [see the magnetic field enhancement in Fig. 5(b)] and satisfies the Kerker condition. By decreasing the distance $l$, the separation between the two modes increases because of the strong near-field coupling.

To understand the underlying physics of the Kerker condition, we note that the proposed structure supports both effective electric and magnetic dipole moments, which are given by (see Appendix D)

$$
\begin{aligned}
& p_{\mathrm{eff}}^{x}=\left(2 p_{1}^{x}+p_{3}^{x}\right) j_{0}(u)+\frac{1}{4}\left(5 p_{1}^{x}-2 p_{3}^{x}\right) j_{2}(u), \\
& m_{\mathrm{eff}}^{y}=\frac{3 i}{2} c\left[\left(p_{1}^{x}-p_{3}^{x}\right) j_{1}(u)+\sqrt{3} p_{2}^{z} j_{2}(u)\right],
\end{aligned}
$$

where $j_{n}(u)$ are spherical Bessel functions, $u=\sqrt{3} k l / 3$, and $c$ is the speed of light in vacuum. $p_{13}^{x}$ and $p_{2}^{z}$ are the electric dipole moments of the individual atoms, along the $x$ and $z$ axes, and are calculated from Eq. (2). Note that in the derivation of Eq. (4), we used $p_{1}^{x}=p_{2}^{x}$ and $p_{1}^{z}=-p_{2}^{z}$ due to the symmetry of the trimer (see Appendix
D). Then, the forward- and backward-scattering cross sections for this atomic antenna can be calculated from (see Appendix D)

$$
C_{\mathrm{sca}}=k^{4}\left|p_{\mathrm{eff}}^{x} \pm m_{\mathrm{eff}}^{y} / c\right|^{2} /\left(4 \pi \epsilon_{0}^{2}\left|\mathbf{E}_{\mathrm{inc}}\right|^{2}\right),
$$

where + and - are used for forward- and backward-scattering cross sections, respectively. Equation (5) shows clearly that the first Kerker condition is fulfilled when $p_{\mathrm{eff}}^{x}=m_{\mathrm{eff}}^{y} / c$. Figure 5(c) plots the real and imaginary parts of the effective electric and magnetic dipole moments. It can be seen that at the Kerker frequency $\omega_{\mathrm{K}}$, both the real and imaginary parts of the induced dipole moments satisfy the Kerker condition independently. Hence, at $\omega_{\mathrm{K}}$, the forward scattering is significantly enhanced, while the backward scattering is considerably suppressed [see Fig. 5(d)]. We note that the generalized Kerker effect with balanced electric dipole and quadrupole moments can be achieved using a 3D atomic antenna (see Appendix E).

A subwavelength ensemble of randomly distributed atoms scatters less than a single atom close to resonance [59]. However, we showed that by arranging the atoms in particular geometries, one can control scattering to achieve extremely small or large scattering cross sections, the so-called scattering dark state and superscattering, respectively. Moreover, we demonstrated that a particular geometry of the atoms fulfills the Kerker condition and scatters light in the forward 
direction. We showed that these geometries exhibit higherorder electric and magnetic multipole moments, although the individual atoms support only electric dipole transitions. We have employed these induced multipole moments to control the scattering. The treatment used in this paper is an excellent approximation to the full quantum model in the weak-excitation regime. Therefore our considerations can also be realized in the quantum regime and pave the way towards the generation and manipulation of photonic quantum states, e.g., realization of single-photon emitters $[60,61]$ and quantum memory $[62,63]$.

\section{ACKNOWLEDGMENTS}

This work was partially supported by the Max Planck Society. R.A. is grateful to Boris Braverman for helpful discussions and acknowledges the support of the Alexander von Humboldt Foundation through the Feodor Lynen Fellowship. R.A., A.S., and R.W.B. gratefully acknowledge support from the Canada First Research Excellence Fund, the Canada Research Chairs program, and the Natural Sciences and Engineering Research Council of Canada (NSERC; Funding Reference No. RGPIN/2017-06880).

\section{APPENDIX A: ATOMIC POLARIZABILITY AND COUPLED-DIPOLE EQUATIONS}

Let us consider an atomic antenna composed of natural atoms with only electric dipole transition moments and illuminated by a plane wave (see Fig. 1). The atoms are arranged at small distances such that the entire size of the antenna is smaller than the wavelength of the light, i.e., $D<\lambda$. We consider the weak-excitation limit where the atomic response is isotropic and linear. The electric polarizability of each atom amounts to $\alpha(\omega)=-\left(\alpha_{0} \Gamma_{0} / 2\right) /\left[\delta+i\left(\Gamma_{0}+\Gamma_{\mathrm{nr}}\right) / 2\right]$, where $\Gamma_{0}$ is the radiative linewidth of the atomic transition at frequency $\omega_{a}, \delta_{a}=\omega-\omega_{a} \ll \omega_{a}$ represents the frequency detuning between the illumination and the atom, and $\alpha_{0}=$ $6 \pi / k^{3}$ ( $k$ is the wave number) [46,47]. We assume elastic scattering events, and therefore the nonradiative decay rate is zero, i.e., $\Gamma_{\mathrm{nr}}=0$. The induced polarization current density for the atomic antenna can be written as

$$
\mathbf{J}(\mathbf{r}, \omega)=-i \omega \sum_{i=1}^{N} \mathbf{p}\left(\mathbf{r}_{i}\right) \delta\left(\mathbf{r}-\mathbf{r}_{i}\right),
$$

where $\delta$ is the Dirac delta function and $\mathbf{p}\left(\mathbf{r}_{i}\right)$ is the induced electric dipole moment of the $i$ th atom placed at $\mathbf{r}=\mathbf{r}_{i}$ (see Fig. 1). In Eq. (A1), we assumed $e^{-i \omega t}$ as a time-harmonic variation. The induced dipole moment of the $i$ th atom $\mathbf{p}\left(\mathbf{r}_{i}\right)=$ $\epsilon_{0} \alpha \mathbf{E}_{\mathrm{loc}}\left(\mathbf{r}_{i}\right)$ can be obtained by using the coupled-dipole equations $[38,52,53]$

$$
\mathbf{p}\left(\mathbf{r}_{i}\right)=\epsilon_{0} \alpha_{i}\left[\mathbf{E}_{\mathrm{inc}}\left(\mathbf{r}_{i}\right)+\sum_{i \neq j} \mathbf{G}\left(\mathbf{r}_{i}, \mathbf{r}_{j}\right) \mathbf{p}\left(\mathbf{r}_{j}\right)\right],
$$

where $\mathbf{E}_{\text {inc }}\left(\mathbf{r}_{i}\right)$ is the incident field at the atom position $\mathbf{r}_{i}$, $\alpha_{i}$ is the atomic polarizability, and $\sum_{i \neq j} \mathbf{G}\left(\mathbf{r}_{i}, \mathbf{r}_{j}\right) \mathbf{p}\left(\mathbf{r}_{j}\right)$ is the interaction field at $\mathbf{r}=\mathbf{r}_{i}$ created by all the other atoms. The total field at the position of the $i$ th atom $\mathbf{E}_{\text {loc }}\left(\mathbf{r}_{i}\right)$ is the sum of the incident field and the scattered field from the other atoms. The electric dipole at position $\mathbf{r}_{j}$ radiates an electromagnetic field which when measured at $\mathbf{r}_{i}$ can be calculated from $\mathbf{G}\left(\mathbf{r}_{i}, \mathbf{r}_{j}\right) \mathbf{p}\left(\mathbf{r}_{j}\right)$, where $\mathbf{G}\left(\mathbf{r}_{i}, \mathbf{r}_{j}\right)$ is Green's tensor and reads as $[54,55]$

$$
\mathbf{G}\left(\mathbf{r}_{i}, \mathbf{r}_{j}\right)=\frac{3}{2 \alpha_{0} \epsilon_{0}} e^{i \zeta}\left[g_{1}(\zeta) \overline{\overline{\mathbf{I}}}+g_{2}(\zeta) \mathbf{n n}\right],
$$

where $\overline{\overline{\mathbf{I}}}$ is the identity dyadic, $\mathbf{n}=\frac{\mathbf{r}_{i}-\mathbf{r}_{j}}{\left|\mathbf{r}_{i}-\mathbf{r}_{j}\right|}$, and $\zeta=$ $\left|k\left(\mathbf{r}_{i}-\mathbf{r}_{j}\right)\right|$. The Cartesian components of Green's tensor are given by

$$
G^{\mu \nu}(\zeta)=\frac{3}{2 \alpha_{0} \epsilon_{0}} e^{i \zeta}\left[g_{1}(\zeta) \delta_{\mu \nu}+g_{2}(\zeta) \frac{\zeta_{\mu} \zeta_{\nu}}{\zeta^{2}}\right],
$$

where

$$
\begin{aligned}
& g_{1}(\zeta)=\left(\frac{1}{\zeta}-\frac{1}{\zeta^{3}}+\frac{i}{\zeta^{2}}\right) \\
& g_{2}(\zeta)=\left(-\frac{1}{\zeta}+\frac{3}{\zeta^{3}}-\frac{3 i}{\zeta^{2}}\right)
\end{aligned}
$$

and where $\mu, v \in x, y, z$.

For atoms with zero nonradiative decay $\Gamma_{\mathrm{nr}}=0$, we get $\operatorname{Im}[1 / \alpha]=-1 / \alpha_{0}$. Using conservation of energy, one can show that the scattered power is equal to the extracted power $P_{\text {sca }}=P_{\text {ext }}$. Thus the scattering cross section and the extinction cross section of the atoms are identical, $C_{\mathrm{sca}}=C_{\mathrm{ext}}$, and the extinction cross section reads as $[38,47,49,53]$

$$
C_{\text {ext }}=\frac{k}{\epsilon_{0}\left|E_{0}\right|^{2}} \sum_{i=1}^{N} \operatorname{Im}\left[\mathbf{p}\left(\mathbf{r}_{i}\right) \cdot \mathbf{E}_{\mathrm{inc}}^{*}\left(\mathbf{r}_{i}\right)\right],
$$

where the extinction cross section $C_{\text {ext }}=P_{\text {ext }} / I_{0}$ is defined as the ratio of the extracted power $P_{\text {ext }}$ to the incident intensity $I_{0}$.

\section{APPENDIX B: SUPERSCATTERING}

In this appendix, we study superscattering in onedimensional and three-dimensional subwavelength atomic antennas.

\section{One-dimensional subwavelength antenna}

Let us consider an atomic pentamer with $N=5$ [see the inset of Fig. 6(a)]. The normalized scattering cross section as a function of frequency detuning $\omega-\omega_{a}$ and distance $l$ is shown in Fig. 6(a). The pentamer exhibits five modes [see the inset of Fig. 6(a)]. $M_{1}$ is a superradiant mode, where all dipoles oscillate in phase, and exhibits a large linewidth compared with the natural linewidth of an isolated atom. In contrast, $M_{5}$ is subradiant with a very narrow linewidth. Figure $6(\mathrm{~b})$ shows the normalized scattering cross section as a function of frequency detuning for $l_{\mathrm{SS}} \approx 0.2 \lambda_{a}$ and the contribution of each multipole moment to the scattering cross section. The maximum scattering cross sections of different multipole moments occur, approximately, at the superscattering frequency $\omega_{\text {Ss }}$. The total scattering cross section is a superposition of the cross sections associated with effective multipole moments. Therefore the scattering cross section of the pentamer far exceeds the scattering cross section of a single atom. Figures 6(c) and 6(d) depict the induced dipole of each atom. At $\omega_{\mathrm{Ss}}$, the induced dipole moment of each 

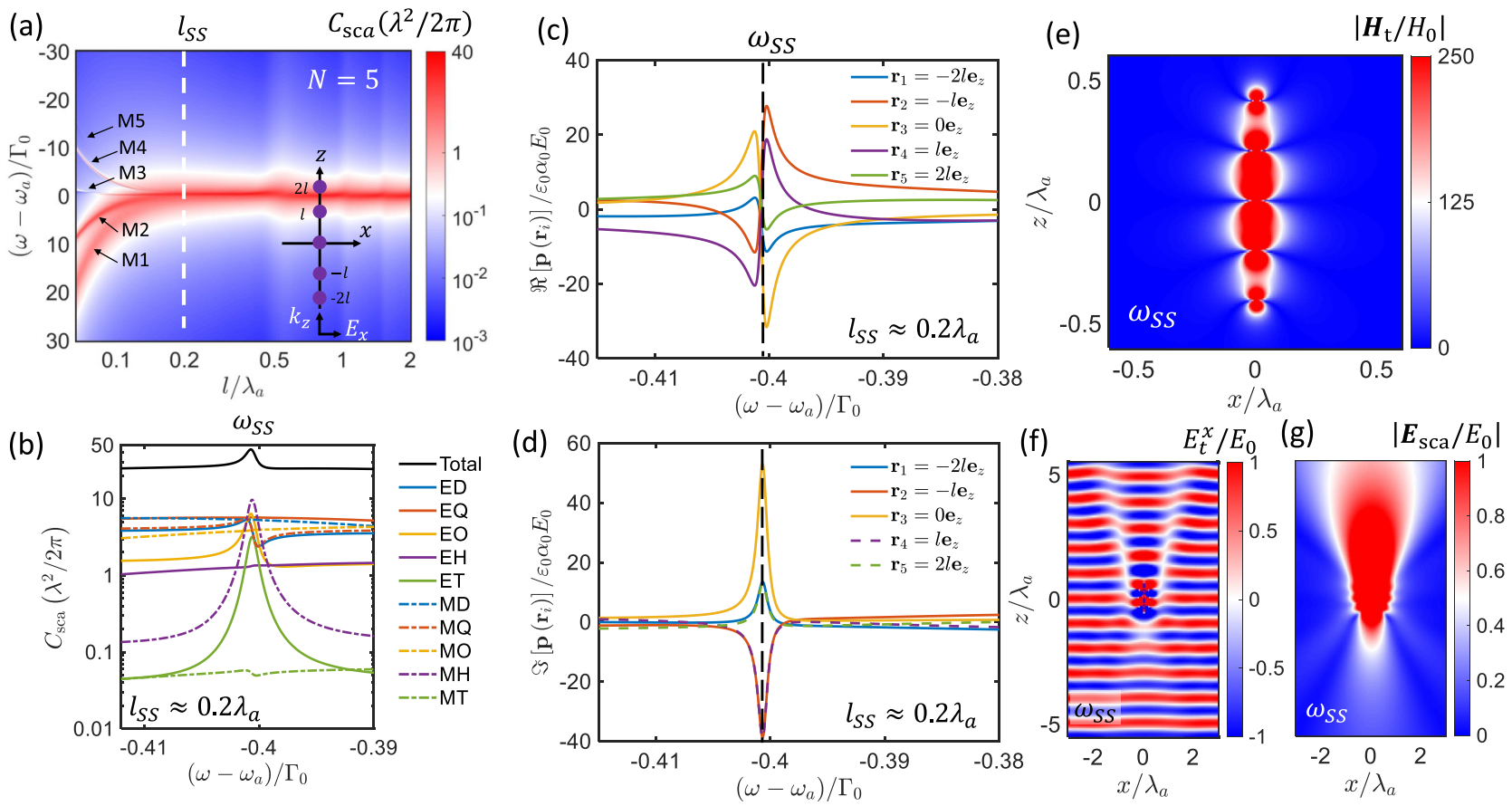

FIG. 6. Superscattering in a pentamer. (a) Scattering cross section normalized to $\lambda^{2} / 2 \pi$ and shown in logarithmic scale. The inset illustrates the atoms placed at $\mathbf{r}_{1,5}=\mp 2 l \mathbf{e}_{z}, \mathbf{r}_{2,4}=\mp l \mathbf{e}_{z}, \mathbf{r}_{3}=0 \mathbf{e}_{z}$. (b) Normalized scattering cross section of different electric and magnetic multipole moments as a function of frequency detuning for $l_{\mathrm{SS}} \approx 0.2 \lambda_{a}$. ED (MD), EQ (MQ), EO (MO), EH (MH), and ET (MT) indicate the electric (magnetic) dipole, quadrupole, octupole, hexadecapole, and triakontadipole moments, respectively. (c) and (d) Real and imaginary parts of the induced dipole moment of each atom as a function of frequency detuning at $l_{\mathrm{SS}}$. (e) Total magnetic field distribution $\mathbf{H}_{t}$ at $\omega_{\mathrm{SS}}$, normalized to the incident magnetic field $H_{0}$. (f) and (g) Real part of the normalized total electric field distribution $E_{t}^{x}$ (the $x$ component) and normalized scattered field distribution $\left|\mathbf{E}_{\mathrm{sca}}\right|$ at $\omega_{\mathrm{sS}}$, respectively.

atom is at resonance. Each dipole is out of phase with respect to neighboring atoms, which explains the strong magnetic field in Fig. 6(e). This strong magnetic field testifies that the pentamer exhibits an induced magnetic multipolar response at $\omega_{\text {ss. }}$. Figures $6(\mathrm{f})$ and $6(\mathrm{~g})$ show the scattered and total (sum of incident and scattered) field distribution at the superscattering frequency $\omega_{\text {Ss }}$. The scattering field is very large even at the far field of the pentamer, and the total field is strongly perturbed. Therefore a higher extinction can be achieved compared with that of a single atom.

\section{Three-dimensional subwavelength antenna}

In the main text, we discussed superscattering from a one-dimensional array of atoms. In this section, we consider superscattering from higher-dimensional subwavelength atomic structures and show that even larger scattering cross sections can be achieved compared with the one-dimensional antenna. Figure 7 shows the scattering cross section normalized to $\lambda^{2} / 2 \pi$ as a function of frequency detuning for different layers of $2 \times 2$ atoms. $N_{s}$ is the number of layers. It can be seen that the scattering cross section of the $3 \mathrm{D}$ structure is significantly larger than that of a single atom, e.g., $C_{\text {sca }}^{\max } \approx$ $28 \times \frac{3 \lambda^{2}}{2 \pi}$ for $N_{s}=4$ although the 3D antenna is composed of $N=16$ atoms (see the inset of Fig. 7).

Figure 8(a) shows a three-dimensional subwavelength antenna composed of $N=12$ atoms, i.e., $N_{s}=3$. The normal- ized scattering cross section of the 3D antenna as a function of frequency detuning and the contribution of each multipole moment to the scattering cross section is shown in Fig. 8(b). The total scattering cross section is a superposition of the cross sections associated with effective multipole moments. At $\omega_{S S}$, the antenna exhibits a strong electric and magnetic

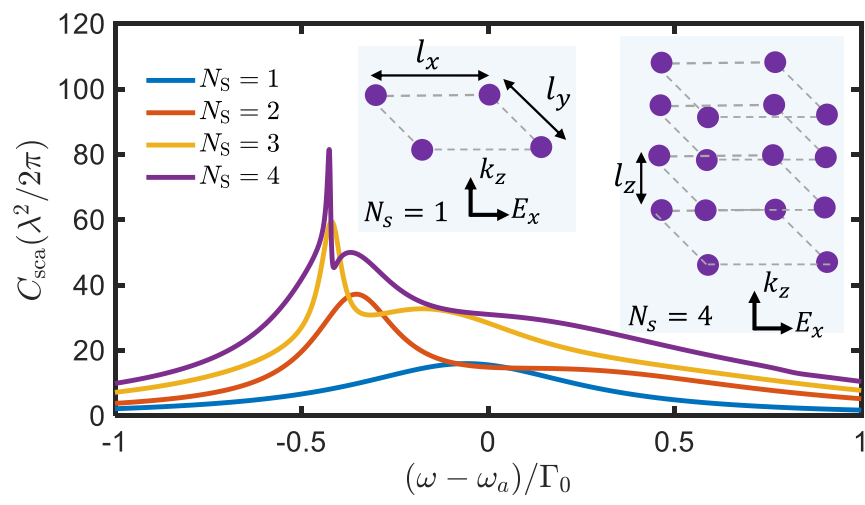

FIG. 7. Superscattering in a three-dimensional subwavelength antenna. Scattering cross section normalized to $\lambda^{2} / 2 \pi$ as a function of frequency detuning $\omega-\omega_{a}$ for different layers of $2 \times 2$ atoms, i.e., $N_{s}=1,2,3,4$ (see insets), where $l_{x}=l_{y}=0.8 \lambda_{a}$ and $l_{z}=0.2 \lambda_{a}$. Note that the size of the antenna is smaller than the wavelength $\left[l_{x}, l_{y},\left(N_{s}-1\right) \times l_{z}<\lambda\right]$. The insets illustrate the three-dimensional subwavelength antenna for $N_{s}=1$ and $N_{s}=4$. 
(a)
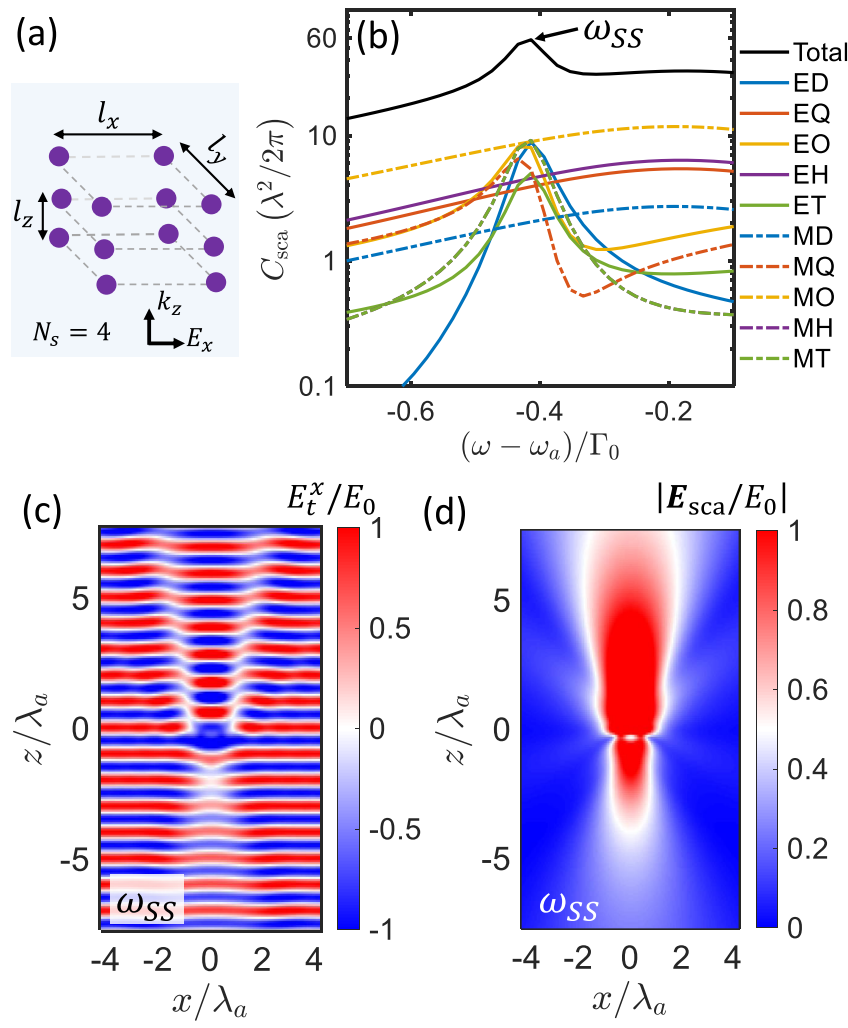

FIG. 8. Superscattering in a three-dimensional subwavelength antenna, $N_{s}=3$. (a) A three-dimensional subwavelength antenna composed of $N=12$ atoms $\left(N_{s}=3\right)$, where $l_{x}=l_{y}=0.8 \lambda_{a}$ and $l_{z}=$ $0.2 \lambda_{a}$. (b) Normalized scattering cross section of different electric and magnetic multipole moments as a function of frequency detuning. ED (MD), EQ (MQ), EO (MO), EH (MH), and ET (MT) indicate the electric (magnetic) dipole, quadrupole, octupole, hexadecapole, and triakontadipole moments, respectively. (c) and (d) Real part of the normalized total electric field distribution $E_{t}^{x}$ (the $x$ component) and normalized scattered field distribution $\left|\mathbf{E}_{\mathrm{sca}}\right|$ at $\omega_{\mathrm{SS}}$, respectively.

response, which leads to a very large scattering cross section $C_{\text {sca }}^{\max } \approx 20 \times \frac{3 \lambda^{2}}{2 \pi}$. Figures $8(\mathrm{c})$ and $8(\mathrm{~d})$ show the scattering and total (sum of incident and scattered, $x$ component) field distribution at the superscattering frequency $\omega_{\mathrm{SS}}$. The scattering field is very large even at the far field, and the total field is strongly perturbed [Fig. 8(c) and 8(d)].

\section{Angular dependence of scattered power}

In this section, we study the angular dependence of scattered power for one-dimensional and three-dimensional subwavelength antennas. Figure 9(a) shows the normalized scattered power as a function of angle in the $x z$ plane at the superscattering frequencies for a one-dimensional subwavelength antenna for different numbers of atoms, $N=2,3,4,5$. By increasing the number of atoms, the backscattering cross section $\left(\theta=180^{\circ}\right)$ decreases for a larger number of atoms. A similar result is shown in Fig. 9(b) for the three-dimensional subwavelength antenna.

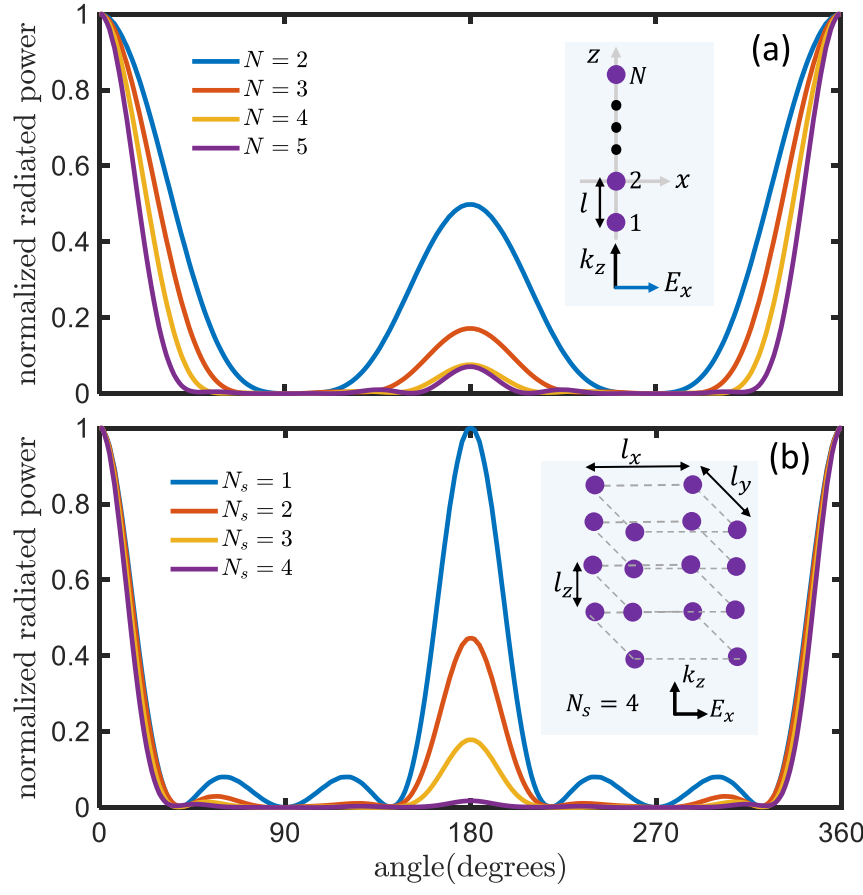

FIG. 9. Normalized scattered power as a function of angle in the $x z$ plane at the superscattering frequencies: (a) one-dimensional subwavelength antenna, where $l=0.2 \lambda_{a}$ and (b) three-dimensional subwavelength antenna, where $l_{x}=l_{y}=0.8 \lambda_{a}$ and $l_{z}=0.2 \lambda_{a}$.

\section{APPENDIX C: SCATTERING DARK STATES}

\section{Atomic trimer}

In this section, we derive effective multipole moments of an atomic trimer and its scattering cross section. The atomic trimer is placed on the $x$ axis with atoms at $\mathbf{r}_{1,3}= \pm l \mathbf{e}_{x}, \mathbf{r}_{2}=$ 0 and is illuminated by an $x$-polarized plane wave propagating in the $z$ direction [see the inset of Fig. 4(a)]. The induced displacement volume current density for the atomic trimer can be written as

$$
\mathbf{J}(\mathbf{r}, \omega)=-i \omega \sum_{i=1}^{3} p_{i}^{x} \delta\left(\mathbf{r}-\mathbf{r}_{i}\right) \mathbf{e}_{x},
$$

where the induced electric dipole moment of each atom can be calculated using the coupled equation [Eq. (A2)] and read as

$$
\begin{aligned}
& p_{1}^{x}=p_{3}^{x}=-\epsilon_{0} \alpha \frac{\epsilon_{0} \alpha \beta_{1}+1}{\epsilon_{0} \alpha\left(2 \epsilon_{0} \alpha \beta_{1}^{2}+\beta_{2}\right)-1} E_{0}, \\
& p_{2}^{x}=\epsilon_{0} \alpha \frac{\epsilon_{0} \alpha\left[\beta_{2}-2 \beta_{1}\right]-1}{\epsilon_{0} \alpha\left(2 \epsilon_{0} \alpha \beta_{1}^{2}+\beta_{2}\right)-1} E_{0},
\end{aligned}
$$

where $p_{1}^{x}=p_{3}^{x}$ due to the symmetry [see the inset of Fig. 4(a)] and $\beta_{1}$ and $\beta_{2}$ are defined by

$$
\begin{aligned}
& \beta_{1} \triangleq G_{12}^{x x}(\zeta=k l)=\frac{3}{2 \alpha_{0} \epsilon_{0}} e^{i k l} g_{1}(k l), \\
& \beta_{2} \triangleq G_{13}^{x x}(\zeta=2 k l)=\frac{3}{2 \alpha_{0} \epsilon_{0}} e^{i 2 k l} g_{1}(2 k l) .
\end{aligned}
$$



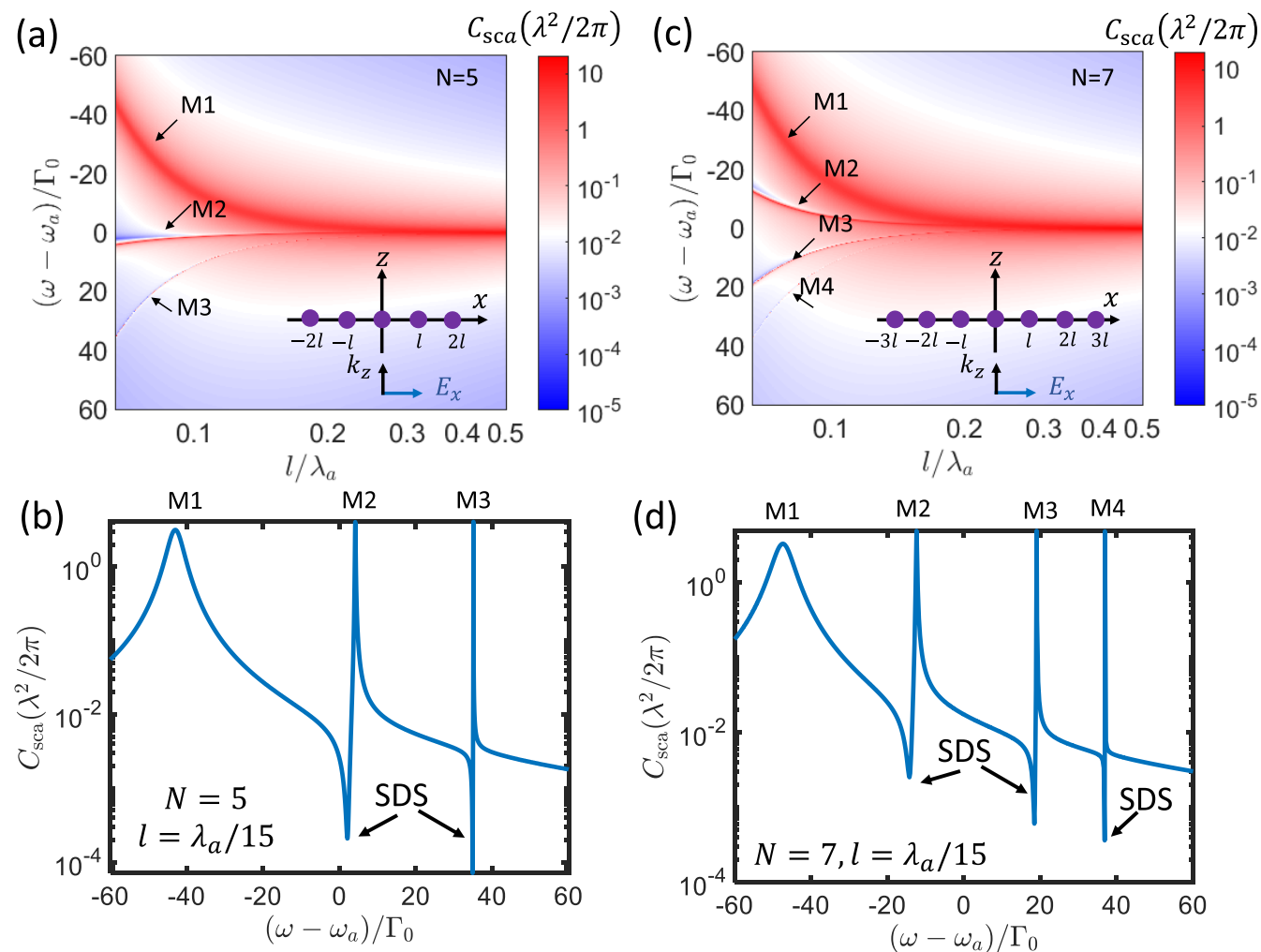

FIG. 10. Scattering dark state as a nonradiating source. (a) and (c) Scattering cross section (normalized to $\lambda^{2} / 2 \pi$ and shown in logarithmic scale) as a function of frequency detuning and the distance between the atoms $l$ for a pentamer $(N=5)$ and a heptamer $(N=7)$, respectively. Insets: schematic drawings of the antenna and the plane-wave excitation. (b) and (d) Normalized scattering cross section as a function of frequency detuning for $l=\lambda_{a} / 15$, for a pentamer and a heptamer, respectively.

Using Eqs. (A6) and (C2), we obtain the scattering cross section of the trimer [i.e., Eq. (3) of the main text]:

$$
\begin{aligned}
C_{\mathrm{sca}} & =C_{\mathrm{ext}}=\frac{k}{\epsilon_{0}\left|E_{0}\right|^{2}} \operatorname{Im}\left[\sum_{i=1}^{3} p_{i} E_{\mathrm{inc}}^{*}\left(\mathbf{r}_{i}\right)\right] \\
& =k \operatorname{Im}\left[\alpha \frac{\epsilon_{0} \alpha\left(\beta_{2}-4 \beta_{1}\right)-3}{\epsilon_{0} \alpha\left(2 \epsilon_{0} \alpha \beta_{1}^{2}+\beta_{2}\right)-1}\right] .
\end{aligned}
$$

Note that for atoms with zero nonradiative decay, the scattering cross section and the extinction cross section of the atomic antenna are identical, $C_{\mathrm{sca}}=C_{\mathrm{ext}}$.

\section{Multipole expansion at the center of the atomic trimer}

One can consider the trimer as a single antenna, effectively. The induced effective electric dipole moment of the trimer can be obtained using the multipole expansion at the center of the trimer, i.e., $\mathbf{r}=0$, and reads as [14-16]

$$
\begin{aligned}
p_{\mathrm{eff}}^{\beta}= & -\frac{1}{i \omega}\left\{\int d v J_{\beta} j_{0}(k r)\right. \\
& \left.+\frac{k^{2}}{2} \int d v\left[3(\mathbf{r} \cdot \mathbf{J}) r_{\beta}-r^{2} J_{\beta}\right] \frac{j_{2}(k r)}{(k r)^{2}}\right\},
\end{aligned}
$$

where $\mathbf{J}$ is the induced displacement volume current in Eq. (C1) and $d v$ is the volume integral $d v=d x d y d z$ in Cartesian coordinates. $\beta=x, y, z$ and $j_{n}(k r)$ are spherical Bessel functions. Now by substituting Eq. (C1) into Eq. (C5), we get

$$
\begin{aligned}
&\left(\mathbf{p}_{\text {eff }}=p_{\text {eff }}^{x} \mathbf{e}_{x}\right) \\
& p_{\text {eff }}^{x}=- \frac{1}{i \omega}\left\{\int J_{x} j_{0}(k r) d v\right. \\
&\left.+\frac{k^{2}}{2} \int d v\left[3(\mathbf{r} \cdot \mathbf{J}) x-r^{2} J_{x}\right] \frac{j_{2}(k r)}{(k r)^{2}}\right\} \\
&=-\frac{1}{i \omega} \int J_{x}\left[j_{0}(k r)+\frac{1}{2}\left(3 \frac{x^{2}}{r^{2}}-1\right) j_{2}(k r)\right] d v \\
&= {\left[p_{1}^{x}+p_{3}^{x}\right]\left[j_{0}(k l)+j_{2}(k l)\right]+p_{2}^{x} } \\
&= \epsilon_{0} \alpha E_{0} \frac{\epsilon_{0} \alpha \beta_{2}-2 \epsilon_{0} \alpha \beta_{1}-1}{\epsilon_{0} \alpha\left[2 \epsilon_{0} \alpha \beta_{1}^{2}+\beta_{2}\right]-1} \\
&-2 \epsilon_{0} \alpha E_{0} \frac{\left(\epsilon_{0} \alpha \beta_{1}+1\right)\left[j_{0}(k l)+j_{2}(k l)\right]}{\epsilon_{0} \alpha\left[2 \epsilon_{0} \alpha \beta_{1}^{2}+\beta_{2}\right]-1} .
\end{aligned}
$$

Note that all the higher-order multipoles are negligible for $k l \ll l$. Using Eq. (A6), we calculate the extinction (scattering) cross section of the trimer

$$
\begin{aligned}
C_{\mathrm{sca}} & =C_{\mathrm{ext}} \approx \frac{k}{\epsilon_{0}\left|E_{0}\right|^{2}} \operatorname{Im}\left[p_{\mathrm{eff}}^{x} E_{\mathrm{inc}}^{*}(\mathbf{r}=0)\right] \\
& \approx k \operatorname{Im}\left[\alpha \frac{\epsilon_{0} \alpha\left(\beta_{2}-4 \beta_{1}\right)-3}{\epsilon_{0} \alpha\left(2 \epsilon_{0} \alpha \beta_{1}^{2}+\beta_{2}\right)-1}\right] .
\end{aligned}
$$

Clearly, Eq. (C7) is identical to Eq. (C4), and one can conclude that the trimer only exhibits an effective electric dipole moment for $k l \ll 1$. In Eq. (C7), we used the long-wavelength 
approximation, i.e., $k l \ll 1$, where the spherical Bessel functions can be approximated by $j_{0}(k l) \approx 1$ and $j_{2}(k l) \approx 0$. Thus the induced electric dipole moment can be written as $\left(p_{\text {eff }}^{x} \approx\right.$ $\left.\epsilon_{0} E_{0} \alpha_{\text {sum }}\right)$

$$
p_{\mathrm{eff}}^{x} \approx \epsilon_{0} \alpha E_{0} \frac{\epsilon_{0} \alpha\left(\beta_{2}-4 \beta_{1}\right)-3}{\epsilon_{0} \alpha\left[2 \epsilon_{0} \alpha \beta_{1}^{2}+\beta_{2}\right]-1} .
$$

\section{Atomic pentamer and heptamer}

Here, we investigate scattering dark states in onedimensional antennas consisting of a larger number of atoms: a pentamer and a heptamer with five and seven atoms, respectively [see the insets of Figs. 10(a) and 10(c)]. The scattering cross sections of the atomic pentamer and heptamer are plotted in Figs. 10(b) and 10(d), respectively. An antenna with $n$ excited modes exhibits $n-1$ scattering dark states [see, e.g., Figs. 10(b) and 10(d)]. For example, the heptamer exhibits four modes with three scattering dark states with vanishing cross sections.

\section{Induced dipole moments}

First, let us start by introducing the Green's function of the atomic trimer [see the inset of Fig. 5(a)]

$$
\begin{aligned}
& \overline{\overline{\mathbf{G}}}_{12}=\left[\begin{array}{ccc}
G_{12}^{x x} & 0 & 0 \\
0 & G_{12}^{y y} & 0 \\
0 & 0 & G_{12}^{z z}
\end{array}\right]=\frac{3}{2 \alpha_{0} \varepsilon_{0}} e^{i k l}\left[\begin{array}{ccc}
g_{1}+\frac{g_{2}}{4} & 0 & 0 \\
0 & g_{1} & 0 \\
0 & 0 & g_{1}
\end{array}\right], \\
& \overline{\overline{\mathbf{G}}}_{13}=\left[\begin{array}{ccc}
G_{13}^{x x} & 0 & G_{13}^{x z} \\
0 & G_{13}^{y y} & 0 \\
G_{13}^{x z} & 0 & G_{13}^{z z}
\end{array}\right]=\frac{3}{2 \alpha_{0} \varepsilon_{0}} e^{i k l}\left[\begin{array}{ccc}
g_{1}+\frac{g_{2}}{4} & 0 & -\frac{\sqrt{3} g_{2}}{4} \\
0 & g_{1} & 0 \\
-\frac{\sqrt{3} g_{2}}{4} & 0 & g_{1}+\frac{3 g_{2}}{4}
\end{array}\right], \\
& \overline{\overline{\mathbf{G}}}_{23}=\left[\begin{array}{ccc}
G_{23}^{x x} & 0 & G_{23}^{x z} \\
0 & G_{23}^{y y} & 0 \\
G_{23}^{x z} & 0 & G_{23}^{z z}
\end{array}\right]=\frac{3}{2 \alpha_{0} \varepsilon_{0}} e^{i k l}\left[\begin{array}{ccc}
g_{1}+\frac{g_{2}}{4} & 0 & \frac{\sqrt{3} g_{2}}{4} \\
0 & g_{1} & 0 \\
\frac{\sqrt{3} g_{2}}{4} & 0 & g_{1}+\frac{3 g_{2}}{4}
\end{array}\right],
\end{aligned}
$$

where $g_{1} \equiv g_{1}(k l)$ and $g_{2} \equiv g_{2}(k l)$ and are given by

$$
\begin{aligned}
& g_{1}(k l)=\left(\frac{1}{k l}-\frac{1}{(k l)^{3}}+\frac{i}{(k l)^{2}}\right), \\
& g_{2}(k l)=\left(-\frac{1}{k l}+\frac{3}{(k l)^{3}}-\frac{3 i}{(k l)^{2}}\right) .
\end{aligned}
$$

Using the coupled-dipole equations [Eq. (A2)] and Green's tensor [Eq. (D2)], we obtain the induced electric dipole moments of each atom:

$$
\begin{aligned}
& p_{1}^{x}=p_{2}^{x}=\varepsilon_{0} \alpha \frac{E_{0}}{D}\left[2 \varepsilon_{0}^{2} \alpha^{2} G_{13}^{x z 2}-\varepsilon_{0} \alpha G_{12}^{y y}-\varepsilon_{0} \alpha G_{13}^{x x}\left(1+\varepsilon_{0} \alpha G_{12}^{y y}\right) e^{i \frac{\sqrt{3}}{2} k l}-1\right] e^{-i \frac{\sqrt{3}}{6} k l}, \\
& p_{3}^{x}=-\varepsilon_{0} \alpha \frac{E_{0}}{D}\left(1+\varepsilon_{0} \alpha G_{12}^{y y}\right)\left[2 \varepsilon_{0} \alpha G_{13}^{x x}+\left(1-\varepsilon_{0} \alpha G_{12}^{x x}\right) e^{i \frac{\sqrt{3}}{2} k l}\right] e^{-i k \frac{\sqrt{3}}{6} l}, \\
& p_{2}^{z}=-p_{1}^{z}=\varepsilon_{0} \alpha \frac{E_{0}}{D}\left\{\varepsilon_{0} \alpha G_{13}^{x z}\left[2 \varepsilon_{0} \alpha G_{13}^{x x}+\left(1-\varepsilon_{0} \alpha G_{12}^{x x}\right) e^{i \frac{\sqrt{3}}{2} k l}\right]\right\} e^{-i k \frac{\sqrt{3}}{6} l},
\end{aligned}
$$




\section{Multipole expansion at the center of the atomic trimer}

Alternatively, one can consider the trimer as an antenna. The induced effective electric dipole moment of the trimer is calculated by applying the multipole expansion at the center of the trimer $\mathbf{r}=0$ using Eq. (C5) and $\mathbf{p}_{\text {eff }}=p_{\text {eff }}^{x} \mathbf{e}_{x}+p_{\text {eff }}^{z} \mathbf{e}_{z}$. Now by substituting Eq. (D1) into Eq. (C5), we get

$$
\begin{aligned}
p_{\mathrm{eff}}^{x}= & -\frac{1}{i \omega} \int J_{x}\left[j_{0}(k r)+\frac{1}{2}\left(3 \frac{x^{2}}{r^{2}}-1\right) j_{2}(k r)\right] d v \\
= & {\left[p_{1}^{x}+p_{2}^{x}+p_{3}^{x}\right] j_{0}\left(\frac{\sqrt{3}}{3} k l\right) } \\
& +\frac{1}{8}\left[5 p_{1}^{x}+5 p_{2}^{x}-4 p_{3}^{x}\right] j_{2}\left(\frac{\sqrt{3}}{3} k l\right) .
\end{aligned}
$$

By using $p_{1}^{x}=p_{2}^{x}$, we get Eq. (4) of the main text:

$$
p_{\mathrm{eff}}^{x}=\left[2 p_{1}^{x}+p_{3}^{x}\right] j_{0}(u)+\frac{1}{4}\left[5 p_{1}^{x}-2 p_{3}^{x}\right] j_{2}(u),
$$

where $u=\frac{\sqrt{3}}{3} k l$. Note that using Eq. (D1) and $p_{1}^{z}=-p_{2}^{z}$, we obtain

$$
p_{\mathrm{eff}}^{z}=-\frac{1}{i \omega} \int J_{z}\left[j_{0}(k r)+\frac{1}{2}\left(3 \frac{z^{2}}{r^{2}}-1\right) j_{2}(k r)\right] d v=0 .
$$

Note that the $y$ component of the electric dipole moment is also zero, i.e., $p_{\text {eff }}^{y}=0$.

The induced effective magnetic dipole moment at the center of the trimer $\mathbf{r}=0$ reads as [14-16]

$$
m_{\mathrm{eff}}^{\beta}=\frac{3}{2} \int d v(\mathbf{r} \times \mathbf{J})_{\beta} \frac{j_{1}(k r)}{k r},
$$

where $\beta=x, y, z$. By substituting Eq. (D1) into Eq. (D7), we obtain

$$
\begin{aligned}
m_{\mathrm{eff}}^{y}= & \frac{3}{2} \int d v(\mathbf{r} \times \mathbf{J})_{y} \frac{j_{1}(k r)}{k r} \\
= & \frac{3}{2 k} \int\left(\frac{z}{r} J_{x}-\frac{x}{r} J_{z}\right) j_{1}(k r) d v \\
= & \frac{3 i}{2} c\left[\frac{p_{1}^{x}}{2}+\frac{p_{2}^{x}}{2}-p_{3}^{x}\right] j_{1}\left(\frac{\sqrt{3}}{3} k l\right) \\
& +i \frac{3 \sqrt{3}}{4} c\left[p_{2}^{z}-p_{1}^{z}\right] j_{2}\left(\frac{\sqrt{3}}{3} k l\right) \\
= & \frac{3 i}{2} c\left[\left(p_{1}^{x}-p_{3}^{x}\right) j_{1}(u)+\sqrt{3} p_{2}^{z} j_{2}(u)\right],
\end{aligned}
$$

which is Eq. (4) of the main text. Note that one can show that the $x$ and $z$ components of the magnetic dipole moments are zero, i.e., $m_{\mathrm{eff}}^{x}=0$ and $m_{\mathrm{eff}}^{z}=0$. The induced multipole moments of the trimer in Fig. 5 of the main text are calculated using Eqs. (D6) and (D8).

\section{APPENDIX E: GENERALIZED KERKER EFFECT}

In this Appendix, we study the generalized Kerker effect in atomic antennas, which occurs among higher-order multipole moments in addition to the dipole moment [5]. In the generalized Kerker effect, the destructive interference between the scattered fields of different multipole moments leads to zero backscattering. To explore this phenomenon in atomic antennas, we study a three-dimensional antenna as shown in
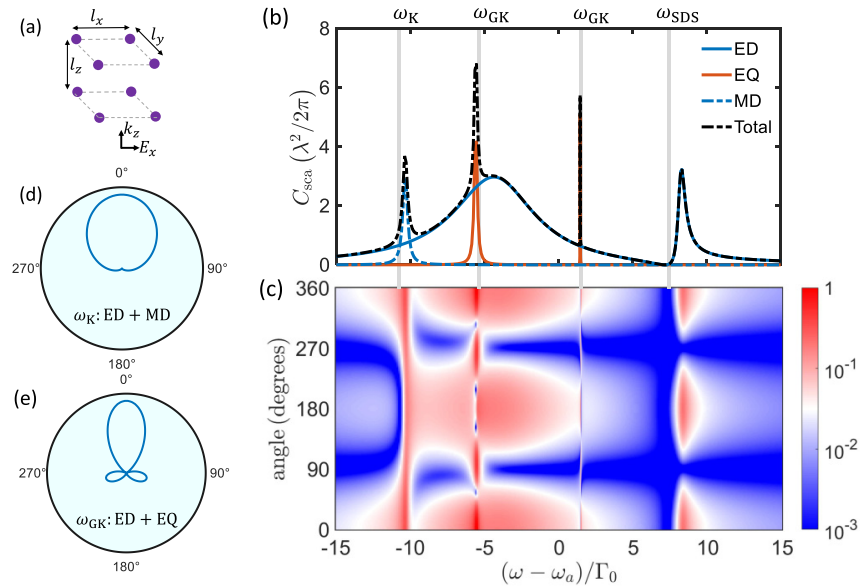

FIG. 11. Generalized Kerker effect. (a) Geometry of an antenna made of eight atoms arranged in subwavelength distances, i.e., $l_{x}=$ $l_{y}=l_{z}=0.1 \lambda_{a}$. (b) Normalized scattering cross section of different electric and magnetic multipole moments as a function of frequency detuning. (c) Normalized scattered power as a function of angle $(x z$ plane) and frequency detuning. (d) The radiation pattern at the first Kerker condition with balanced electric and magnetic dipole moments. (e) The radiation pattern at the generalized Kerker condition with balanced electric dipole and quadrupole moments.

Fig. 11(a). The normalized scattering cross section of the 3D antenna and the angular dependence of scattered power are shown in Figs. 11(b) and 11(c), respectively. Remarkably, the antenna exhibits different scattering phenomena at different frequencies: (i) the Kerker effect at $\omega_{\mathrm{K}}$ [balanced electric and magnetic dipole moments; see Figs. 11(b) and 11(d)], (ii) the generalized Kerker effect at $\omega_{\mathrm{GK}}$ [balanced electric dipole and quadrupole moments; see Figs. 11(b) and 11(e)], and (iii) a scattering dark state at $\omega_{\text {SDS }}$ [vanishing electric and magnetic multipole moments; see Fig. 11(b)]. Note that the generalized Kerker effect occurs at two different frequency detunings.

\section{APPENDIX F: RADIATION PATTERN}

Having the induced multipole moments of the trimer, i.e., Eqs. (D6) and (D8), the far field of an atomic antenna can be found using $[5,55]$

$$
\begin{aligned}
\mathbf{E}_{\mathrm{ED}} & =\frac{k^{2}}{4 \pi \epsilon_{0}} \frac{e^{i k r}}{r} p_{x}\left(-\sin \varphi \mathbf{e}_{\varphi}+\cos \theta \cos \varphi \mathbf{e}_{\theta}\right), \\
\mathbf{E}_{\mathrm{MD}} & =\frac{k^{2}}{4 \pi \epsilon_{0}} \frac{e^{i k r}}{r} \frac{m_{y}}{c}\left(-\cos \theta \sin \varphi \mathbf{e}_{\varphi}+\cos \varphi \mathbf{e}_{\theta}\right), \\
\mathbf{E}_{\mathrm{EQ}} & =\frac{k^{2}}{4 \pi \epsilon_{0}} \frac{e^{i k r}}{r} \frac{i k}{6} Q_{z x}^{e}\left[\cos \theta \sin \varphi \mathbf{e}_{\varphi}-\cos 2 \theta \cos \varphi \mathbf{e}_{\theta}\right], \\
\mathbf{E}_{\mathrm{MQ}} & =\frac{k^{2}}{4 \pi \epsilon_{0}} \frac{e^{i k r}}{r} \frac{i k}{6 c} Q_{z y}^{m}\left[\cos 2 \theta \sin \varphi \mathbf{e}_{\varphi}-\cos \theta \cos \varphi \mathbf{e}_{\theta}\right],
\end{aligned}
$$

where $r, \theta$, and $\varphi$ are the radial distance, polar angle, and azimuthal angle, respectively. The radiation pattern $\left(\propto|\mathbf{E}|^{2}\right)$ in the $x z$ plane, i.e., $\varphi=0$, considering the contribution from all multipole moments (up to magnetic quadrupole), can be 
calculated using the electric field at the far field

$$
\begin{aligned}
\mathbf{E} \approx & \frac{k^{2}}{4 \pi \epsilon_{0}} \frac{e^{i k r}}{r}\left\{p_{x} \cos \theta+\frac{m_{y}}{c}-\frac{i k}{6} Q_{x z}^{e}\left(2 \cos ^{2} \theta-1\right)\right. \\
& \left.-\frac{i k}{6 c} Q_{z y}^{m} \cos \theta\right\} \mathbf{e}_{\theta} .
\end{aligned}
$$

For an atomic trimer with $\lambda \gg l$ at the $M_{2}$ mode, i.e., $\omega_{\mathrm{K}}$, the electric and magnetic quadruple moments are negligible, i.e., $Q_{x z}^{e} \approx 0$ and $Q_{y z}^{m} \approx 0$. Thus the radiation pattern $\left(\propto|\mathbf{E}|^{2}\right)$ in Fig. 5(d) is mainly due to the electric and magnetic dipole moments, and the electric field at the far field reads as

$$
\mathbf{E} \approx \frac{k^{2}}{4 \pi \epsilon_{0}} \frac{e^{i k r}}{r}\left[p_{x} \cos \theta+\frac{m_{y}}{c}\right] \mathbf{e}_{\theta},
$$

where $p_{x}$ and $m_{y}$ for the atomic trimer in Fig. 5 can be obtained using Eqs. (D6) and (D8), respectively.
[1] C. F. Bohren and D. R. Huffman, Absorption and Scattering of Light by Small Particles (John Wiley, New York, 2008).

[2] V. Sandoghdar, M. Agio, X.-W. Chen, S. Götzinger, and K.-G. Lee, Antennas, quantum optics and near-field microscopy, in Optical Antennas, edited by M. Agio and A. Alù (Cambridge University Press, Cambridge, 2013), pp. 100-121.

[3] M. Kerker, D.-S. Wang, and C. L. Giles, Electromagnetic scattering by magnetic spheres, J. Opt. Soc. Am. 73, 765 (1983).

[4] X. Zambrana-Puyalto, I. Fernandez-Corbaton, M. L. Juan, X. Vidal, and G. Molina-Terriza, Duality symmetry and Kerker conditions, Opt. Lett. 38, 1857 (2013).

[5] R. Alaee, R. Filter, D. Lehr, F. Lederer, and C. Rockstuhl, A generalized Kerker condition for highly directive nanoantennas, Opt. Lett. 40, 2645 (2015).

[6] W. Liu and Y. S. Kivshar, Generalized Kerker effects in nanophotonics and meta-optics, Opt. Express 26, 13085 (2018).

[7] A. J. Devaney and E. Wolf, Radiating and nonradiating classical current distributions and the fields they generate, Phys. Rev. D 8, 1044 (1973).

[8] G. Gbur and E. Wolf, Nonradiating sources and other "invisible" objects, Prog. Opt. 45, 273 (2003).

[9] C. W. Hsu, B. G. DeLacy, S. G. Johnson, J. D. Joannopoulos, and M. Soljacic, Theoretical criteria for scattering dark states in nanostructured particles, Nano Lett. 14, 2783 (2014).

[10] Z. Ruan and S. Fan, Superscattering of Light from Subwavelength Nanostructures, Phys. Rev. Lett. 105, 013901 (2010).

[11] Z. Ruan and S. Fan, Design of subwavelength superscattering nanospheres, Appl. Phys. Lett. 98, 043101 (2011).

[12] C. Qian, X. Lin, Y. Yang, X. Xiong, H. Wang, E. Li, I. Kaminer, B. Zhang, and H. Chen, Experimental Observation of Superscattering, Phys. Rev. Lett. 122, 063901 (2019).

[13] A. Krasnok, D. Baranov, H. Li, M.-A. Miri, F. Monticone, and A. Alú, Anomalies in light scattering, Adv. Opt. Photonics 11, 892 (2019).

[14] I. Fernandez-Corbaton, S. Nanz, R. Alaee, and C. Rockstuhl, Exact dipolar moments of a localized electric current distribution, Opt. Express 23, 33044 (2015).

[15] R. Alaee, C. Rockstuhl, and I. Fernandez-Corbaton, An electromagnetic multipole expansion beyond the long-wavelength approximation, Opt. Commun. 407, 17 (2018).

[16] R. Alaee, C. Rockstuhl, and I. Fernandez-Corbaton, Exact multipolar decompositions with applications in nanophotonics, Adv. Opt. Mater. 7, 1800783 (2019).

[17] R. Alaee, D. Lehr, R. Filter, F. Lederer, E.-B. Kley, C. Rockstuhl, and A. Tünnermann, Scattering dark states in multiresonant concentric plasmonic nanorings, ACS Photonics 2, 1085 (2015).
[18] S. D. Jenkins and J. Ruostekoski, Controlled manipulation of light by cooperative response of atoms in an optical lattice, Phys. Rev. A 86, 031602(R) (2012).

[19] W. Feng, Y. Li, and S.-Y. Zhu, Cooperative spontaneous emission of three identical atoms, Phys. Rev. A 88, 033856 (2013).

[20] V. E. Lembessis, A. A. Rsheed, O. M. Aldossary, and Z. Ficek, Two-atom system as a nanoantenna for mode switching and light routing, Phys. Rev. A 88, 053814 (2013).

[21] R. J. Bettles, S. A. Gardiner, and C. S. Adams, Cooperative ordering in lattices of interacting two-level dipoles, Phys. Rev. A 92, 063822 (2015).

[22] R. J. Bettles, S. A. Gardiner, and C. S. Adams, Cooperative eigenmodes and scattering in one-dimensional atomic arrays, Phys. Rev. A 94, 043844 (2016).

[23] R. J. Bettles, S. A. Gardiner, and C. S. Adams, Enhanced Optical Cross Section via Collective Coupling of Atomic Dipoles in a 2D Array, Phys. Rev. Lett. 116, 103602 (2016).

[24] G. Facchinetti, S. D. Jenkins, and J. Ruostekoski, Storing Light with Subradiant Correlations in Arrays of Atoms, Phys. Rev. Lett. 117, 243601 (2016).

[25] A. Asenjo-Garcia, M. Moreno-Cardoner, A. Albrecht, H. J. Kimble, and D. E. Chang, Exponential Improvement in Photon Storage Fidelities Using Subradiance and "Selective Radiance" in Atomic Arrays, Phys. Rev. X 7, 031024 (2017).

[26] E. Shahmoon, D. S. Wild, M. D. Lukin, and S. F. Yelin, Cooperative Resonances in Light Scattering from Two-Dimensional Atomic Arrays, Phys. Rev. Lett. 118, 113601 (2017).

[27] D. Barredo, V. Lienhard, S. De Leseleuc, T. Lahaye, and A. Browaeys, Synthetic three-dimensional atomic structures assembled atom by atom, Nature (London) 561, 79 (2018).

[28] D. S. Wild, E. Shahmoon, S. F. Yelin, and M. D. Lukin, Quantum Nonlinear Optics in Atomically Thin Materials, Phys. Rev. Lett. 121, 123606 (2018).

[29] G. Facchinetti and J. Ruostekoski, Interaction of light with planar lattices of atoms: Reflection, transmission, and cooperative magnetometry, Phys. Rev. A 97, 023833 (2018).

[30] R. J. Bettles, M. D. Lee, S. A. Gardiner, and J. Ruostekoski, Quantum and nonlinear effects in light transmitted through planar atomic arrays, Commun. Phys. 3, 141 (2020).

[31] P.-O. Guimond, A. Grankin, D. V. Vasilyev, B. Vermersch, and P. Zoller, Subradiant Bell States in Distant Atomic Arrays, Phys. Rev. Lett. 122, 093601 (2019).

[32] O. Černotík, A. Dantan, and C. Genes, Cavity Quantum Electrodynamics with Frequency-Dependent Reflectors, Phys. Rev. Lett. 122, 243601 (2019).

[33] A. Grankin, P. O. Guimond, D. V. Vasilyev, B. Vermersch, and P. Zoller, Free-space photonic quantum link and chiral quantum optics, Phys. Rev. A 98, 043825 (2018). 
[34] V. Mkhitaryan, L. Meng, A. Marini, and F. J. G. de Abajo, Lasing and Amplification from Two-Dimensional Atom Arrays, Phys. Rev. Lett. 121, 163602 (2018).

[35] V. Sandoghdar, Nano-optics in $2020 \pm 20$, Nano Lett. 20, 4721 (2020).

[36] R. Bekenstein, I. Pikovski, H. Pichler, E. Shahmoon, S. F. Yelin, and M. D. Lukin, Quantum metasurfaces with atom arrays, Nat. Phys. 16, 676 (2020).

[37] J. Rui, D. Wei, A. Rubio-Abadal, S. Hollerith, J. Zeiher, D. M. Stamper-Kurn, C. Gross, and I. Bloch, A subradiant optical mirror formed by a single structured atomic layer, Nature $\mathbf{5 8 3}$, 369 (2020).

[38] R. Alaee, B. Gurlek, M. Albooyeh, D. Martín-Cano, and V. Sandoghdar, Quantum Metamaterials with Magnetic Response at Optical Frequencies, Phys. Rev. Lett. 125, 063601 (2020).

[39] K. E. Ballantine and J. Ruostekoski, Optical Magnetism and Huygens' Surfaces in Arrays of Atoms Induced by Cooperative Responses, Phys. Rev. Lett. 125, 143604 (2020).

[40] A. Browaeys and T. Lahaye, Many-body physics with individually controlled Rydberg atoms, Nat. Phys. 16, 132 (2020).

[41] A. S. Solntsev, G. S. Agarwal, and Y. S. Kivshar, Metasurfaces for quantum photonics, arXiv:2007.14722.

[42] R. H. Dicke, Coherence in spontaneous radiation processes, Phys. Rev. 93, 99 (1954).

[43] R. H. Lehmberg, Radiation from an $N$-atom system. I. General formalism, Phys. Rev. A 2, 883 (1970).

[44] R. Friedberg, S. R. Hartmann, and J. T. Manassah, Frequency shifts in emission and absorption by resonant systems ot twolevel atoms, Phys. Rep. 7, 101 (1973).

[45] M. Gross and S. Haroche, Superradiance: An essay on the theory of collective spontaneous emission, Phys. Rep. 93, 301 (1982).

[46] Ad. Lagendijk and B. A. Van Tiggelen, Resonant multiple scattering of light, Phys. Rep. 270, 143 (1996).

[47] P. De Vries, D. V. Van Coevorden, and A. Lagendijk, Point scatterers for classical waves, Rev. Mod. Phys. 70, 447 (1998).

[48] G. Zumofen, N. M. Mojarad, V. Sandoghdar, and M. Agio, Perfect Reflection of Light by an Oscillating Dipole, Phys. Rev. Lett. 101, 180404 (2008).

[49] R. Alaee, M. Albooyeh, and C. Rockstuhl, Theory of metasurface based perfect absorbers, J. Phys. D: Appl. Phys. 50, 503002 (2017).
[50] P. Lambropoulos and D. Petrosyan, Fundamentals of Quantum Optics and Quantum Information (Springer, Berlin, 2007).

[51] P. W. Milonni, R. Loudon, P. R. Berman, and S. M. Barnett, Linear polarizabilities of two- and three-level atoms, Phys. Rev. A 77, 043835 (2008).

[52] L. L. Foldy, The multiple scattering of waves. I. General theory of isotropic scattering by randomly distributed scatterers, Phys. Rev. 67, 107 (1945).

[53] G. W. Mulholland, C. F. Bohren, and K. A. Fuller, Light scattering by agglomerates: Coupled electric and magnetic dipole method, Langmuir 10, 2533 (1994).

[54] C.-T. Tai, Dyadic Green Functions in Electromagnetic Theory, IEEE Series on Electromagnetic Waves (Oxford University Press, New York, 1994).

[55] J. D. Jackson, Classical Electrodynamics (Wiley, New York, 1999).

[56] C. J. Foot, Atomic Physics, Oxford Master Series in Physics, Vol. 7 (Oxford University Press, New York, 2005).

[57] G. Wrigge, I. Gerhardt, J. Hwang, G. Zumofen, and V. Sandoghdar, Efficient coupling of photons to a single molecule and the observation of its resonance fluorescence, Nat. Phys. 4, 60 (2008).

[58] R. F. Ribeiro, L. A. Martínez-Martínez, M. Du, J. CamposGonzalez-Angulo, and J. Yuen-Zhou, Polariton chemistry: Controlling molecular dynamics with optical cavities, Chem. Sci. 9, 6325 (2018).

[59] N. J. Schilder, C. Sauvan, Y. R. P. Sortais, A. Browaeys, and J.-J. Greffet, Near-Resonant Light Scattering by a Subwavelength Ensemble of Identical Atoms, Phys. Rev. Lett. 124, 073403 (2020).

[60] C. W. Chou, S. V. Polyakov, A. Kuzmich, and H. J. Kimble, Single-Photon Generation from Stored Excitation in an Atomic Ensemble, Phys. Rev. Lett. 92, 213601 (2004).

[61] A. T. Black, J. K. Thompson, and V. Vuletić, On-Demand Superradiant Conversion of Atomic Spin Gratings into Single Photons with High Efficiency, Phys. Rev. Lett. 95, 133601 (2005).

[62] L.-M. Duan, M. D. Lukin, J. I. Cirac, and P. Zoller, Longdistance quantum communication with atomic ensembles and linear optics, Nature (London) 414, 413 (2001).

[63] R. Reimann, W. Alt, T. Kampschulte, T. Macha, L. Ratschbacher, N. Thau, S. Yoon, and D. Meschede, CavityModified Collective Rayleigh Scattering of Two Atoms, Phys. Rev. Lett. 114, 023601 (2015). 